\section{GRP-127 ON-LINE QUALITY CONTROL OF CYTOTOXIC DRUGS: ULTRA-FAST CHROMATOGRAPHIC SEPARATION OF VINCA ALKALOIDS}

doi:10.1136/ejhpharm-2013-000276.127

'E Caudron, 'E Majoul, 'P Prognon, 'L Havard, 2P Chaminade. 'Hopital Européen Georges Pompidou, Pharmacy, Paris, France; ${ }^{2}$ Univ. Paris Sud, Groupe de chimie Analytique de Paris Sud, Châtenay-Malabry, France

Background Due to repetitive and tedious handling tasks, production of anticancer drugs for infusion is associated with a high risk of non-conformity. Thus, on-line quality control is necessary to improve the quality of preparation. Since the quantities produced are ever growing, very fast analytical methods of control are needed to minimise the delay before release.

Purpose A high-performance liquid chromatography method has been developed for quality control of vinca alkaloid infusion bags (vindesine, vincristine, vinorelbine and vinblastine).

Materials and Methods The separation was optimised by a Doehlert experimental design using a mixture of those 4 alkaloids. Chromatography was performed using Prostar Varian chromatographic equipment with a Photodiode array Detector. A short Polaris C18 column $(3 \mu \mathrm{m}, 50 \mathrm{~mm} \times 4.6 \mathrm{~mm})$ was used for all separations. The optimization varied 3 parameters: $\mathrm{pH}$ of the phosphate buffer $25 \mathrm{mM}$ (7.0-7.6), flow rate of the mobile phase (0.7-1.3 mL. $\left.\mathrm{min}^{-1}\right)$ and proportion of acetonitrile (47-53\%). 36 trials were necessary. The target response was the shortest run time giving a minimal resolution score of 1.5 for the most critical pair of peaks.

Results For vinorelbine, $\mathrm{pH}$ had a major effect on resolution. Optimal resolutions were obtained with a $\mathrm{pH}$ of 7.25 . Then, the flow rate was set at $1.6 \mathrm{~mL} \cdot \mathrm{min}^{-1}$ with a mobile phase consisting of water-acetonitrile $(47-53 \mathrm{v} / \mathrm{v})$. Under these conditions, resolution was at least 1.6 with an analysis time less than 2.0 min. Retention times were 1.03, 1.27, 1.39 and 1.68 minutes for vindesine, vincristine, vinblastine and vinorelbine respectively. Methods were validated according to ICH criteria and are now routinely used without troubleshooting.

Conclusions This method allows in-line quality control of 4 vinca alkaloids in a very short time (less than 2 minutes) and constitutes a suitable and safe tool for chemotherapy preparation.

No conflict of interest.

\section{GRP-128 PARENTERAL IRON: FRENCH PHARMACISTSÍ CHOICES FOR PREVENTING IATROGENIC EVENTS}

doi:10.1136/ejhpharm-2013-000276.128

J Dos-Reis, J Oliary, A Magassa, DT Hoang, C Fernandez, H Barreteau. Groupe Hospitalier Lariboisière - Fernand Widal, Pharmacie, Paris Cedex 10, France

Background Ferric carboxymaltose (FCM) (Ferinject) is a new parenteral iron used to treat iron deficiency anaemia. Infusion modalities are different from ferric hydroxide sucrose complex (FHSC), formerly used in our hospital until July 2012. In particular, for FCM, the infusion takes $15 \mathrm{~min}$ (or less at doses below $500 \mathrm{mg}$ ), instead of $90 \mathrm{~min}$ for FHSC (for which shorter infusion times may be dangerous). Despite the higher cost of FCM and the lack of recommendations from our National Health Agency, we have decided, to avoid medicines errors, to keep Ferinject only on the hospital formulary.

Purpose To discover the approach followed by other Parisian Public Assistance Hospitals (AP-HP) $(n=40)$ to avoid the risk of confusion between the different forms of parenteral iron.

Materials and Methods In October 2012, a questionnaire was developed to assess 3 items: the type of parenteral iron on the hospital formulary, methods of dispensing and occurrence of medicines errors. Hospital pharmacists were phoned to answer the survey.

Results Of the 30 pharmacists who responded, 14 referenced FCM and 16 did not; 11/14 stocked both FCM and FHSC. Three pharmacists had opted to keep FCM only. In $50 \%$ of hospitals in which FCM was available, it had to be prescribed by the brand name and checked by a pharmacist. Two hospitals of the 14 had reserved FCM for specific wards. To the best of their knowledge of pharmacists who answered the survey, no iron administration error had occurred in their hospitals.

Conclusions This study shows that choice of parenteral iron is not homogeneous in the different hospitals of AP-HP. We suggest that the risk of medication errors, when FCM and FHSC are both present in the same hospital, could be underestimated.

No conflict of interest.

\section{GRP-129 PARENTERAL NUTRITION-ASSOCIATED CHOLESTASIS}

doi:10.1136/ejhpharm-2013-000276.129

A Hernández-Sánchez, A Gil-Martin, P Tejada-Gonzalez, T Molina-García. Hospital Universitario de Getafe, Pharmacy, Madrid (Getafe), Spain

Background Parenteral nutrition-associated cholestasis (PNAC) results in significant morbidity and mortality. Progression to endstage liver disease and subsequent hepatic failure is the most feared complication. A number of approaches have been proposed for the prevention and treatment of PNAC with mixed results.

Purpose To investigate the alteration of liver blood tests and the parenteral nutrition (PN) characteristics that trigger PNAC.

Materials and Methods Clinical blood tests and PN data of adults on artificial nutrition from January to August 2012 were collected.

Survival studies were conducted for each liver parameter studied. Primary endpoint was to fall above the upper limit of normal, considering them for women and men respectively: aspartate transaminase (ASP): 32, $40 \mathrm{IU} / \mathrm{L}$; Alanine transaminase (ALT): 78, $78 \mathrm{IU} / \mathrm{L}$; gamma-glutamyl transferase (GGT): 55, 85 IU/L; alkaline phosphatase (ALP): 136, 129 IU/L; bilirubin: <1, <1 mg/dl.

PASW Statistics 19.0 and Microsoft Office 2007 were used.

Results One thousand eight hundred and ten PN bags for 124 patients (55\% men) with mean 61 years old (18-95) were analysed.

Percentage of patients with values within limits after follow-up bilirubin 92\%; ALT 76\%; ASP 59\%; ALP 54\%; GGT 27\%.

Time until values went out of normal limits (days): ALT (13); ALP (13); ASP (12); bilirubin (12); GGT (6).

Age, gender, liver enzymes value before $\mathrm{PN}$, and $\mathrm{PN}$ characteristics (volume, timing of infusions, calories, nitrogen and carbohydrates) were not significant PNAC trigger factors when considered individually.

Risk factor: initial value of bilirubin (each $0.1 \mathrm{mg} / \mathrm{dL}$ before PN, multiplies the risk of hyperbilirubinaemia by 14.5 times).

Protective factor: PN fat content (each gramme reduces the risk of high serum GGT concentration by $3.6 \%$ ).

Conclusions The results show that $\mathrm{PN}$ poses a risk factor for PNAC, GGT being the test most affected.

However, none of the factors surrounding the $\mathrm{PN}$ and the patient, individually, account for the majority of the liver damage. On the contrary it is a conglomerate of different factors contributing to the final impairment. The lack of enteral nutrition also predisposes to PNAC.

This makes it difficult to find the right approach when prescribing PN. The indications for PN should be considered responsibly as should a return to enteral feeding whenever possible.

No conflict of interest. 